\title{
Mobile Image Analysis for Microscopic Images of Seeds
}

\author{
$\mathrm{Ke} \mathrm{Gao}^{1}$, Michele Warmund ${ }^{2}$, Tommi White ${ }^{3}$, Ruthie Angelovici ${ }^{4}$, and Filiz Bunyak ${ }^{1}$
}

1. Department of Electrical Engineering and Computer Science, University of Missouri-Columbia, MO, USA

2. Division of Plant Sciences, University of Missouri-Columbia, MO, USA

3. Department of Biochemistry, University of Missouri-Columbia, MO, USA

4. Division of Biological Sciences, University of Missouri-Columbia, MO, USA

Seeds sustain the beginning stage of a plant [1]. Seed kernel size and shape are crucial because they represent one of the major components of yield [2]. Therefore, it is important to develop an accurate, lowcost, and high-throughput morphometry method that allows for a detailed analysis of seed samples for the purpose of testing plant yield and vigor. Traditional methods of measuring individual seed morphometry or counting seeds in a cluster involve heavy manual work [3]. They have been proven to be labor-intensive, slow and inconsistent. Several tools that are based on image analysis have been developed to accelerate the measuring process, but it still requires a considerable length of time for sample preparation since the seeds need to be carefully placed either on a tray or a scanner in most cases. For accuracy, seeds should not be touching each other during the measurement. Ensuring so is particularly hard for small seeds such as Arabidopsis seeds.

This work presents an image processing application named MUSeed that automatically computes seed morphometry. First, K-means clustering is used to segment the seeds from the background. Mathematical morphology operations are performed to refine the segmentation. After connected components are labelled, clusters consisting of multiple touching seeds are identified based on size and shape criteria for further processing by the cluster decomposition module. Accurate segmentation of individual seeds within a cluster is crucial for morphometry operations. While small clusters of 2-3 seeds can be resolved with simple approaches, decomposition of larger clusters are challenging. For robust and accurate cluster decomposition, candidate segmentations are generated with complementary region-based (Watershed with Edge-Augmented Markers) and boundary-based (Concave Point Analysis) systems. The final segmentation is determined with a novel automated evaluation and selection scheme that operates on multi-scale and multi-method outputs. The selection process ensures robustness under different seed arrangements where reliable region or boundary information may not be available. Finally, shape and size parameters are automatically computed for the individual seeds identified by the segmentation and cluster decomposition modules. Figure 1(a) shows an Arabidopsis seeds sample imaged using mobile microscope. Area of each individual seed is approximately $0.1 \mathrm{~mm}^{2}$. As shown in the image, several challenges can be observed such as uneven illumination, noise, and touching seeds. Figure 1(b) demonstrates the segmentation result generated by the proposed method. All the seeds are correctly located and accurately segmented even for the clusters where multiple seeds are touching each other. Contour as well as major axis of each individual seed is marked.

Based on the proposed seed segmentation algorithm, an Android-based mobile application (MUSeed) for seed morphometry has been developed. The application is implemented in Java with OpenCV libraries. The integrated development environment is Android Studio. A seed image can be captured either by taking a picture using the smartphone camera or by loading an existing image saved in the phone. The application is capable of processing seed images and generating measurements of each seed. A general quantitative 
analysis on the whole set of seeds in the image can be presented to the user by showing the total count, average area, average perimeter, average width, average length, etc. In addition, the individual seed measurement is also available. The measurements can be exported to a text file and sent to an email address provided by the user.

\section{References:}

[1] Moore, Candace R., et al. "Mapping quantitative trait loci affecting Arabidopsis thaliana seed morphology features extracted computationally from images." G3: Genes| Genomes| Genetics 3.1 (2013): 109-118.

[2] Alex P Whan, Alison B Smith, Colin R Cavanagh, Jean-Philippe F Ral, Lindsay M Shaw, Crispin A Howitt, and Leanne Bischof, "Grainscan: a low cost, fast method for grain size and colour easurements," Plant methods, vol. 10, no. 1, pp. 1-10, 2014.

[3] Ramya, P., et al. "QTL mapping of 1000-kernel weight, kernel length, and kernel width in bread wheat (Triticum aestivum L.)." Journal of applied genetics 51.4 (2010): 421-429.

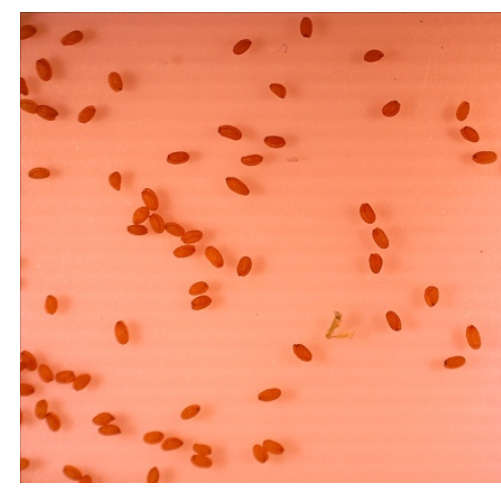

(a)

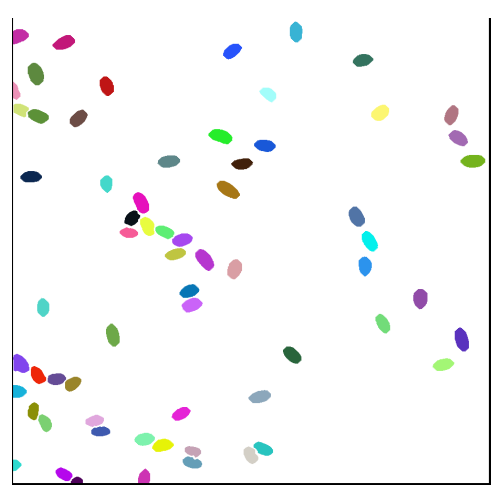

(b)

Figure 1. (a) Sample Arabidopsis seeds imaged using mobile microscope. (b) Seed segmentation result from the proposed method.
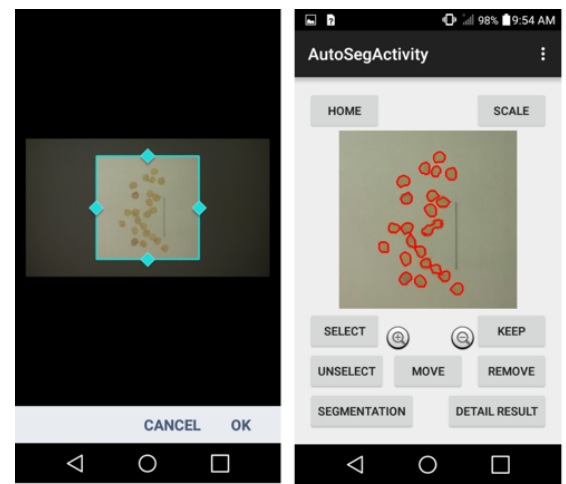

(a)

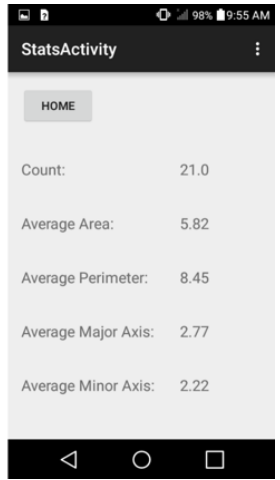

$\triangleleft \quad 0 \quad \square$

Figure 2. (a) Major graphical user interfaces of MUSeed. (b) MUSeed pipeline.

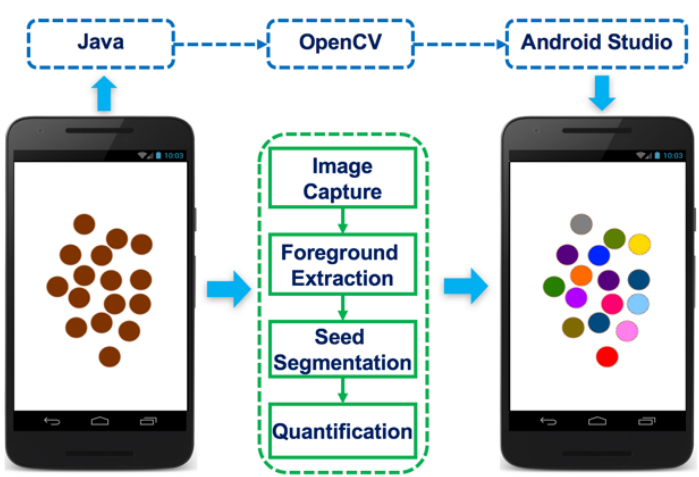

(b) 